\title{
The Orderly Charging and Discharging Dispatching Management System of Electric Vehicle under the Internet
}

\author{
Yuman Zhang \\ School of North China Electric Power University, Beijing 102206, China
}

291229717@qq.com

Keywords: Internet; Electric vehicle; Charge-discharge; Dispatch; Network platform

\begin{abstract}
The orderly charging and discharging management and dispatching system of electric vehicle is researched and designed based on internet in order to achieve orderly dispatching and management of electric vehicle in peak load regulation. Researching and analyzing the function of main components (power grid side, platform data center, platform management system, charging pile and client) of system and designing the hardware and software of the system. To optimize the data processing part of backstage network server, combining with the security and economy of grid and the maximization of owner benefit by using multi-objective and multi-constraint optimization model. System can realize bidirectional communication and real-time update regulation and control function among grid side, system backstage and client through internet and protect the liability and high efficiency of electric vehicle in grid-connected dispatching.
\end{abstract}

\section{Introduction}

In recent years, with the development of electric vehicle scale and the coming age of internet and big data, under the "Internet plus" pattern, the widespread use of electric vehicle has driven the development of global electric traffic internet and the global electric vehicle will also become the important basis of global traffic internet. In the internet, intelligent processing and high-efficient transfer in information can develop networking of charge-discharge technology for electric vehicles. In addition to realize cleaning and energy-saving transport, an increasing number of electric vehicles also show that they can be able to provide reliable and high-quality power supply to the power grid.

For the technology development of electric vehicle in peak load regulation, the international society puts forward the policy of V2G (Vehicle-to-Grid) in recent years which takes the electric vehicle as movable distributed energy storage device and feeds back the electric energy to power grid under the premise of satisfying the driving demand of the users of electric vehicle. The current studies show that it is a challenge to dispatch electric vehicles in grid connection. Domestic and overseas studies mainly concentrate on relevant applications of $\mathrm{V} 2 \mathrm{G}$, frequency modulation, optimal unit combination, as well as costs and benefits of the networked electric vehicles in economy or technology, and influences on the environment. However, reliability and operability of electric vehicles' power connection can be affected by distribution uncontrollability of electric vehicles and subjectivity of owners to a great extent. As a result, it will be of great importance in studying the organic combination of the networked electric vehicles and the internet and orderly charge-discharge management strategy of electric vehicles in practical applications.

Based on the existing grid-connected technology of electric vehicles, the author studied power grid parameters and user demands under different sub-regions and provided a kind of internet-oriented charge-discharge control and management strategy, which realizes peak load regulation of electric vehicles via two-way communication of the internet and webpage platform.

\section{System Composition}

The whole system consist of four parts which are network platform, charging pile, power grid side and client. 
Network platform as a network server is a integrated platform for data collection, storage, processing and maintenance of electric vehicle charging and discharging. And it consists of computer, network equipment, storage equipment, and other peripheral devices and platform application software. Network platform is the core of the whole system which can collect power grid information, release charging and discharging plan, calculate and operate regularly and also can operate and implement the workflow.

Charging pile is a charging device for electric vehicle. The charging pile can monitor the state of electric vehicle real-timely and share the information with server and it also is a direct link between network platform and client.

Client as user side is an interface for owner to participant in electricity management. Users can inquire the information of the power grid load demand, electricity price forecasting and the place and state of charging pile through web platform and also they can make confirmation to participant in grid connection from client and make a series of operations to make money, such as charge in low and discharge in peak.[1]

Communication relationships among the four are as follows:

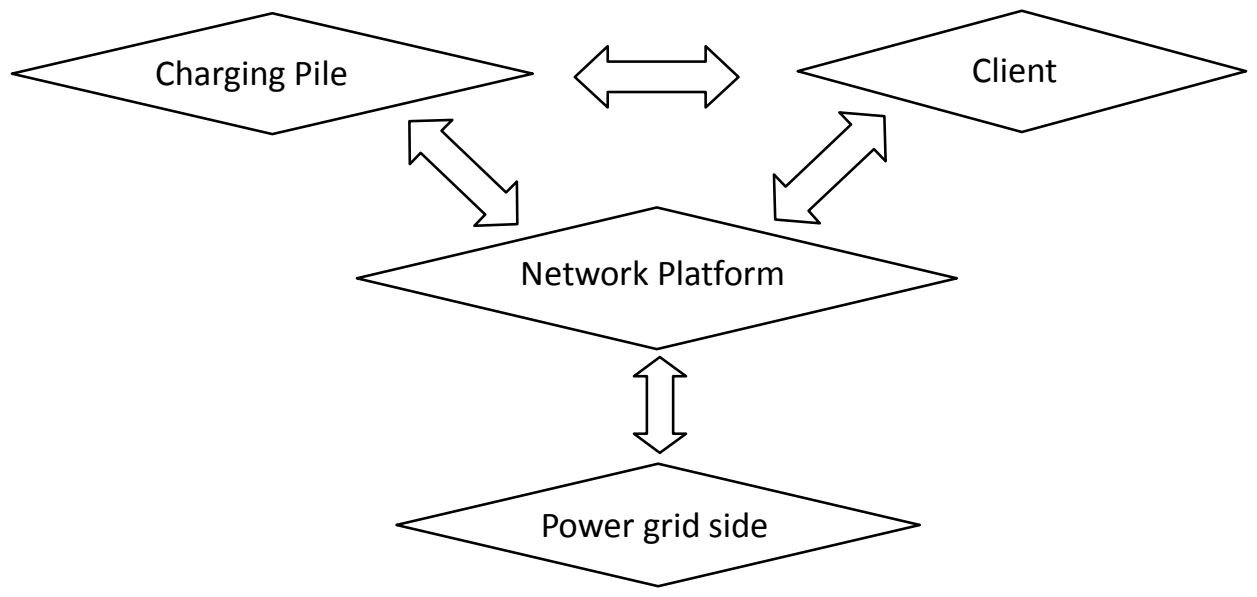

\section{Overall Design Scheme}

During the operational process of the whole system, the network platform will be divided into data center and management system specifically. The data of power grid real-time frequency, power grid load forecasting and the state prediction of $\mathrm{V} 2 \mathrm{G}$ will be sent to platform data center from power grid side according to the peak load regulation demand information. Data center deals with these data through relevant algorithm strategy and on the premise of protecting the power grid security and users interest, makes a plan of $\mathrm{V} 2 \mathrm{G}$ charging and discharging and sends the charging and discharging plan and each order to platform management system. After accepting the plan, management sends information to charging pile and client in order to release demand time, demand for electricity quantity and price forecasting and at the same time management system feeds back the electricity quantity of electric vehicle to platform through charging pile and feeds back the top and bottom limit of charging participation to the platform through users. Users as the direct connection between charging pile and data center feeds back the charging and discharging running condition of charging pile and electric vehicle to data center real-timely in order to update and adjust the plan and thus reach the bidirectional communication. Charging pile reasonably chooses vehicle distribution and the charging and discharging power in order to guarantee the execution of the charging and discharging. Users choose whether to participate in grid connection under the condition of ensuring the orderly discharging of vehicles and the information of the real-time electricity quantity also be verified by charging pile and thus get the permission of battery charging and discharging. The specific operation chart is shown in fig. 1. 


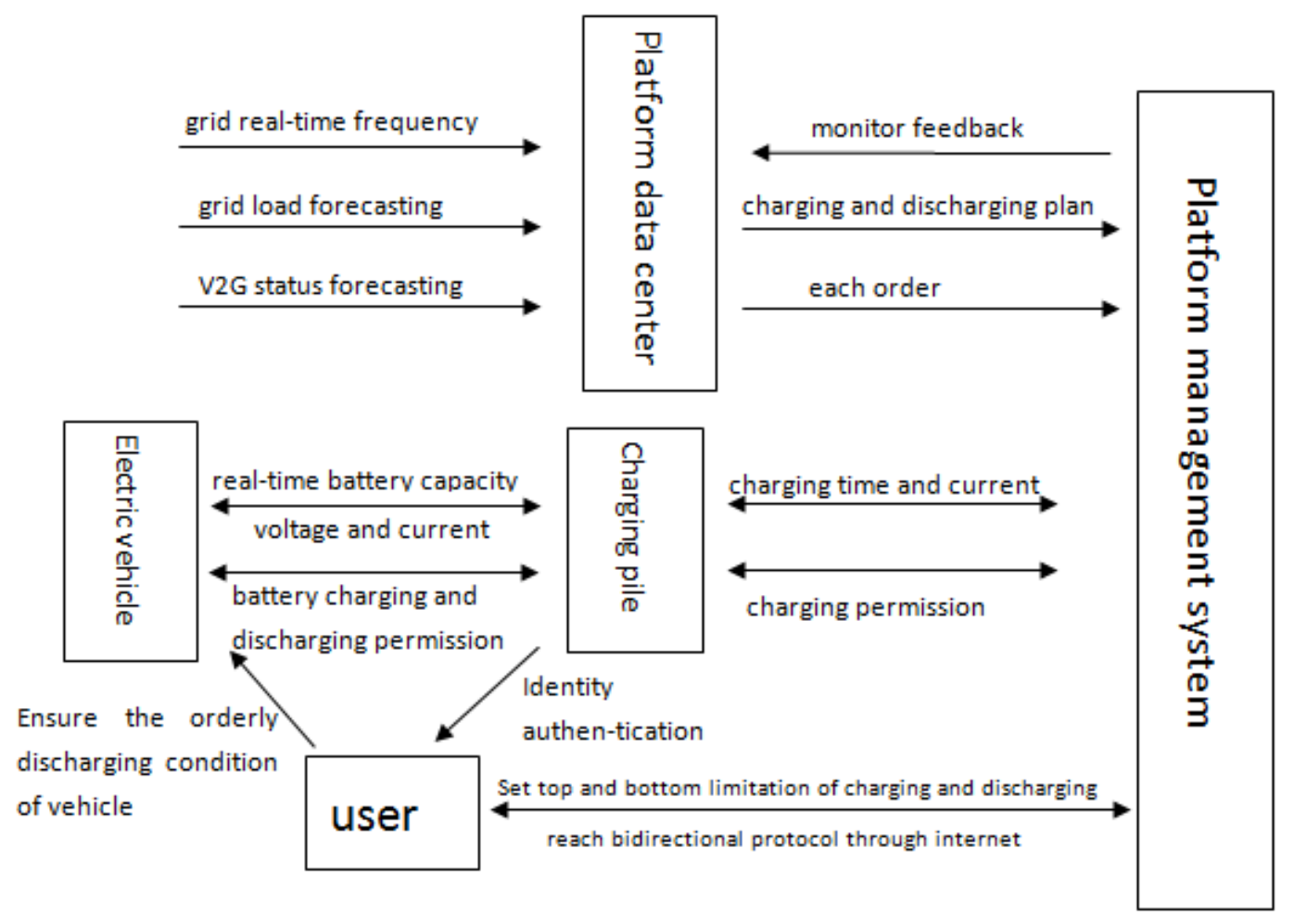

Figure 1. System run chart

System Hardware Design. The main hardware of system basically consists of three parts. The background control center which consists of platform database and background server, the data transfer unit which consists of concentrator and wireless devices and field collection device which consists of charging pile as a main body. The specific schematic diagram as shown in fig. 2.[2]

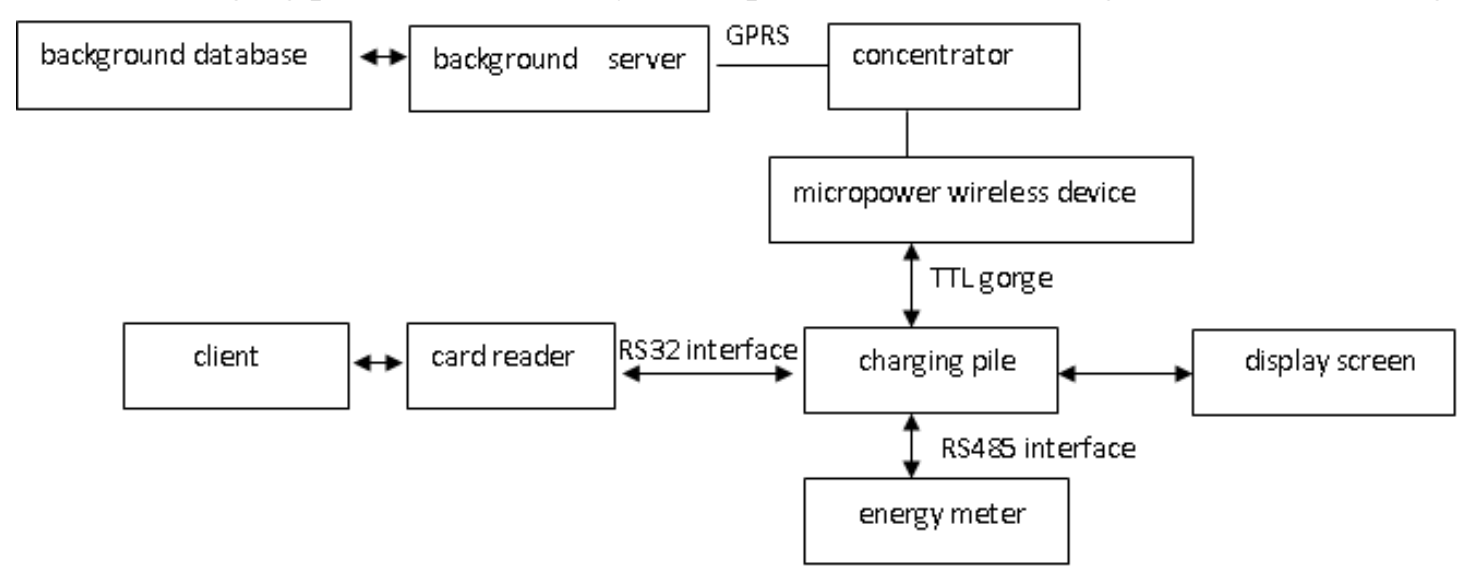

Figure 2. System hardware structure diagram

The unit circuit design of whole control system is as follows:

(1)Master Controller

A master controller selects a PLC controller, which has a series of functions, including logical operation, timing, technology, displacement, self-diagnosis and monitoring, as well as a little analog quantity $\mathrm{I} / \mathrm{O}$, arithmetic operation, data transmission, remote $\mathrm{I} / \mathrm{O}$ and communication. As a result, the controller was selected as the core control unit of the hardware system to launch, operate, monitor and close the charging process in real time [3].

(2)Serial Interface Circuits

In the system, four serial interfaces are connected with display screen, wireless module, RS232 interface's card reader and RS485 interface's electric energy meter. The display is RS232 level and 
communicates with MCU through level switch. The communication protocol of display screen and MCU is the Modbus RTU communication protocol. MCU is used as the master, while display screen is the slave. The card reader belongs to TTL level and it can be connected with MCU directly. Moreover, the protocol proposed by the card reader module is applied to communicate. TTL serial port is used in micropower wireless module. Level 2.0 electric energy meter is selected as the electric energy meter for battery charging measurement [4], which has the current specification of 5A. The processor PCL realizes information interaction between electric energy measuring modules through RS485 bus interface circuits, which are shown in Fig. 3. Energy metering module abides by the DL/T645-2007 communication specification. Electric energy value on the meter can be used as the electric energy measurement of the charging piles. At the same time, current and voltage on the ammeter can be used to judge whether there is overcurrent, overvoltage and undervoltage in the charge-discharge process. If so, relevant measures should be applied to deal with it[5].

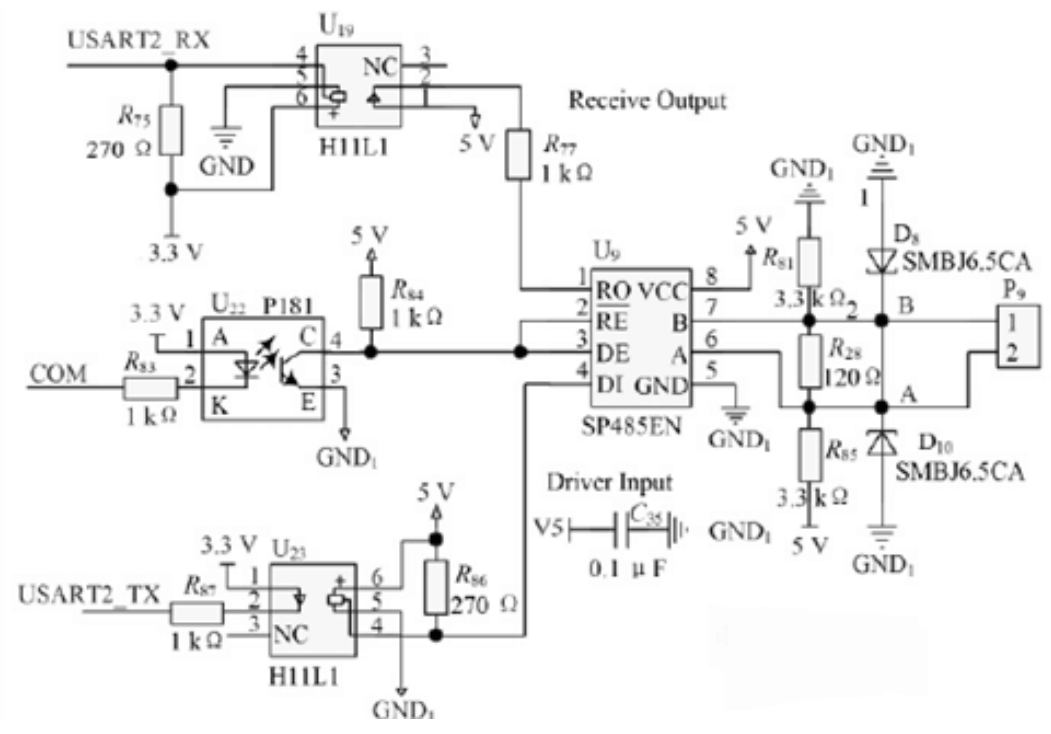

Figure 3. RS485 interface circuit diagram

(3) Control Guiding Circuits

The confirmative connection circuits between charging piles and charging cables, as well as control guiding circuits between charging piles and vehicle-mounted battery chargers are able to control the guiding circuits to finish connective confirmation of charging piles and electric vehicles before charging, power, identification of current-carrying capability for charge-discharge connecting devices, as well as monitoring of the charge-discharge process. The circuits are preconditions for charging piles, battery chargers and battery management system to realize information exchange. With the different voltage values on detection points, MCU is used to judge the state and the interface circuits are shown in Fig. 4. Generally speaking, the circuits should be equipped with stable output of $+12 \mathrm{~V}$ and bipolar PWM signal functions. In the PWM state, peak and valley should be $+12 \mathrm{~V}$ and $-12 \mathrm{~V}$, respectively. By virtue of PWM pin of PLC, amplifying circuits Q1 and Q2 are amplified and outputted from Cp after 6N135 isolation, where R7 is the output matched resistance. According to GB/T20234.2[6], resistance should be $1000 \Omega$. R5 has the metering function and protects LED[7]. 


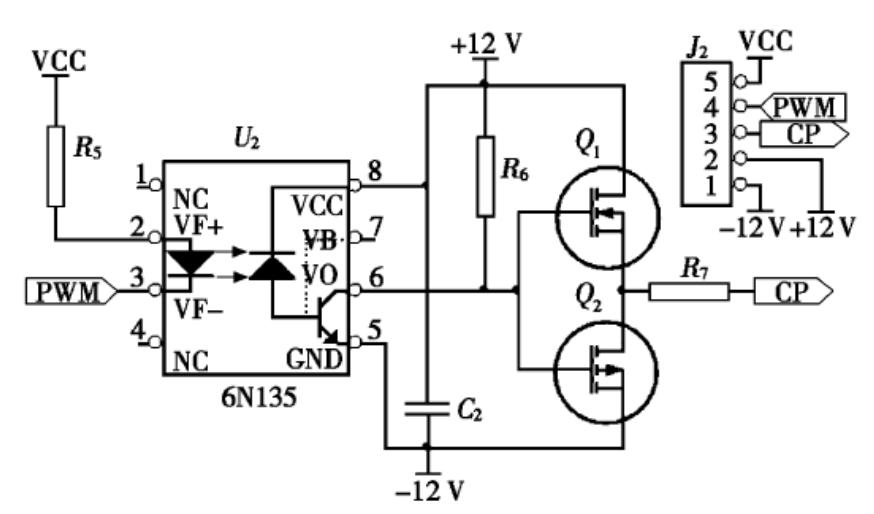

Figure 4. Control Guiding Circuits diagram

System Software Design. System program mainly consists of system initialization, system self-inspection, swiping card effective identification, user validation, system connection validation and the real-time monitoring during charging process. Realize reading user information and settlement at the same time in the part of user authentication. In the part of real-time monitor during charging process, mainly collecting real-timely and analyzing and processing the $\mathrm{CP}$ signal in order to ensure the connection status of charging cable, the situation of surplus electric quantity and device state. Charging pile completes interaction control through display screen. Charging and discharging model offers multiple choices. The charging and discharging can be set according to time, electric quantity and amount of money and also can be set to full directly or on the minimum value. Charging process is similar to discharging process. Now list the overall flow chart of the charging program which as shown in fig. 5. 


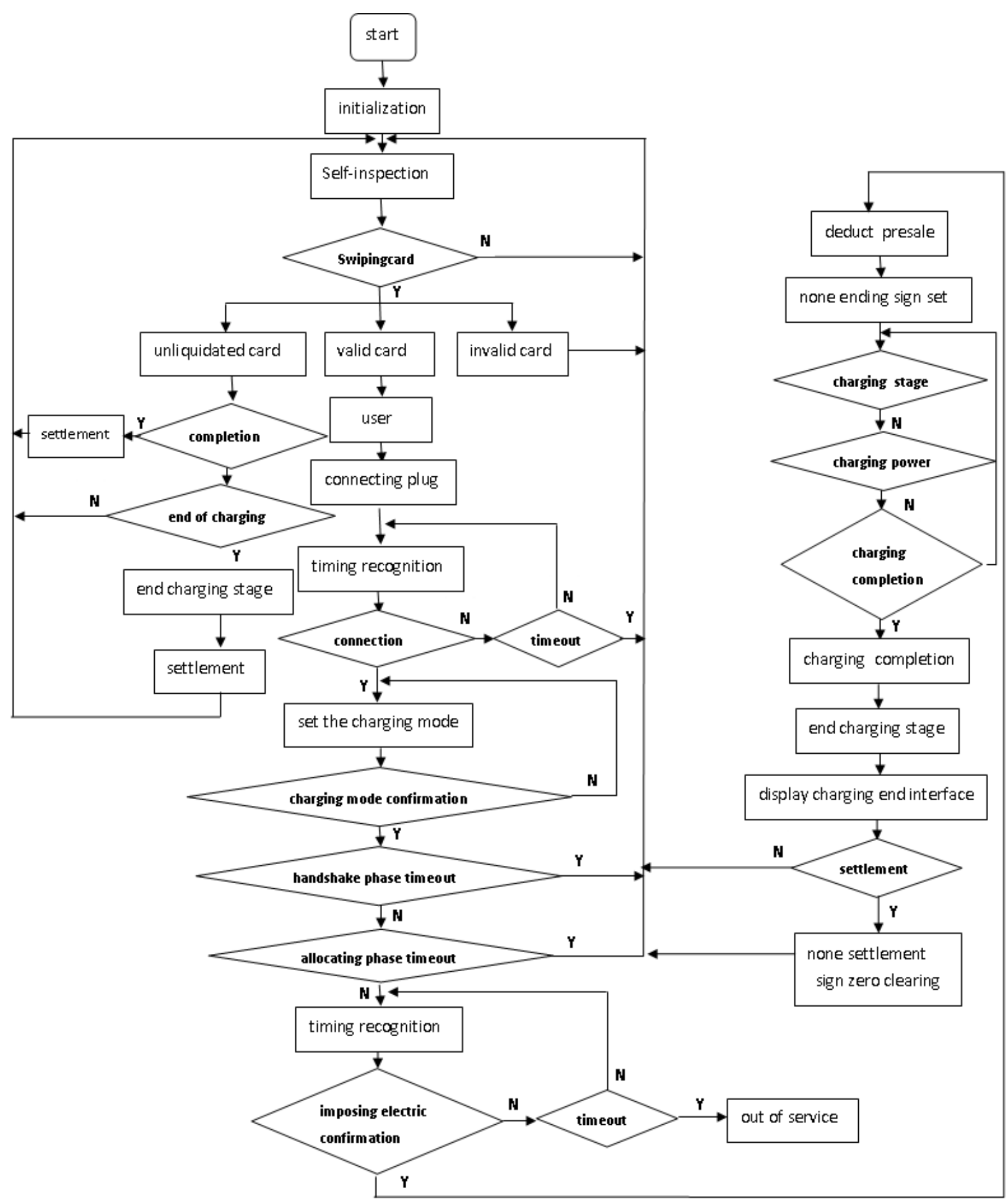

Figure 5. Software design flow chart

Rule Design. System internal procedure and external management execute the command according to certain rules which are divided into pricing rule, user participation rule, information processing rule and information release rule.

1. Pricing Rule

Charge capacity not only can execute tariff but also can participate in electric power transaction directly and buy low charge capacity. The electric quantity of charging can be used by themselves and also can be sold to power grid as distributed power. Release the price forecasting to users through web platform.

2. User Participation Rule 
Users participate in power grid peak shaving and valley filling through active selection and are encouraged to participate by the market mechanism which caused by the electricity price difference. Users can obtain the information of price, discharge capacity and charging pile distribution in web platform and also can choose the participation discharge capacity and participation time. Once the users confirm to participate and input data in web platform they cannot exit the charging and discharging plan. The users' action will be regulated by the users credit line mechanism in the web platform. The user flow chart is shown in fig. 6[8].

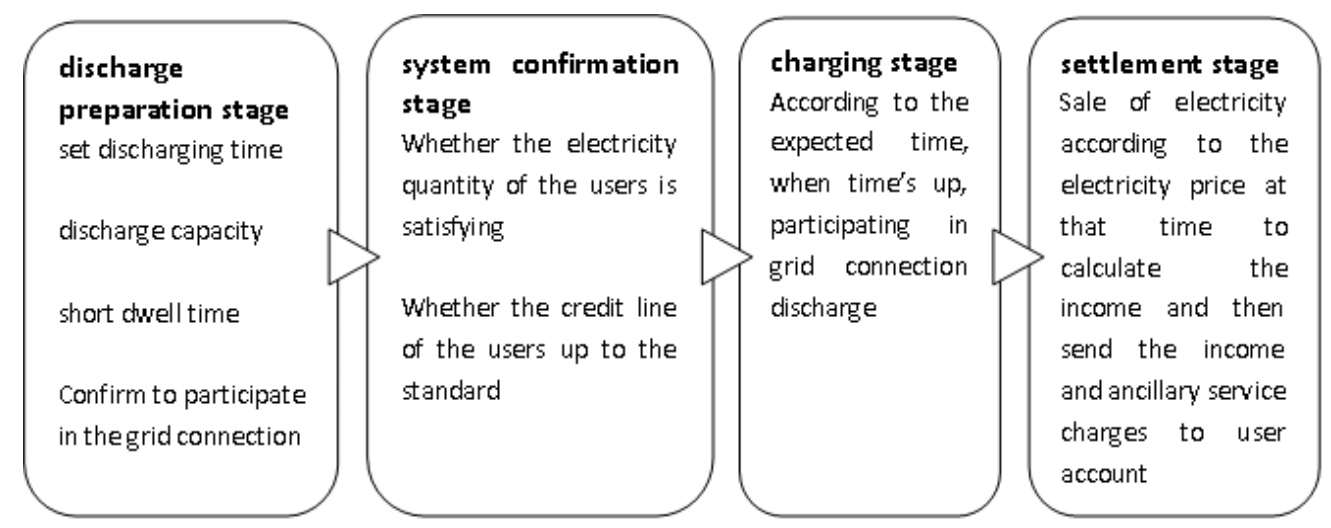

Figure 6. The flow chart of user charging

\section{Information Processing Rule}

Processing the information collected by power grid is the important gist to make charging and discharging plan in network data center. Multi-objective optimization model is adopted as the processing rule because of the randomness of charging and discharging behavior of the owner in order to make electric vehicle charge and discharge reasonably and satisfy the driving demand of electric vehicle and the economic interest of the owner at the same time of protecting the safe and economical operation of power grid. This paper only gives the confirmation of objective function and the making of constraints condition as a calculation basis for reference. [9]

(1) Objective Function

Synthetically considering the common interests of power grid side and owner, four objective functions are established from the perspective of power grid side and owner.

1) Minimal node voltage deviation and active loss of power grid

Based on load prediction data of power grid, power flow equation is used to calculate.

$$
\min f_{1}=\sum_{t=1}^{N_{1}}\left[\sum_{i=1}^{N_{2}} \Delta U_{i}(t)^{2}\right]
$$

$\min f_{2}=\sum_{t=1}^{N_{1}} P(t)$

In the formula: $\Delta U_{i}(t)$ is the voltage excursion of note $i$ at $t, P(t)$ is the power grid loss at $t$; $N_{1}$ is the charge-discharge periods in a day; $N_{2}$ is node number.

2) Lowest networked service costs

Power grid is used to provide V2G state prediction. A cost function is listed as follows

$$
\min f_{3}=\sum\left[\operatorname{pri}_{v 1}(t) P_{V}(t) \Delta t\right]
$$


In the formula: $\operatorname{pri}_{v 1}(t)$ is electricity price to servicers paid by power grid as networking vehicles at $t ; P_{V}(t)$ is the input power from electric vehicles to power grid at $t ; t$ is the discharge time.

3) Maximal benefits of owners

From the perspective of owners, predictive data of electricity price is used. Charging costs should be reduced, for the sake of ensuring maximal economic benefits for owners, namely the charge cost function is shown as follows:

$$
\min f_{4}=\sum_{t=1}^{N_{1}}\left[p r i_{g}(t) \mathrm{P}_{G}(t) \Delta t-p r i_{v 2}(t) \mathrm{P}_{V}(t) \Delta t\right]
$$

In the formula: ${ }^{p r i} i_{g}(t)$ is the favorable charge price for owners given by power grid at $t$; $\operatorname{pri}_{v 2}(t)$ represents the electricity price from servicers of vehicle networking to owners at $t ; \mathrm{P}_{G}(t)$ means the charge power of electric vehicles at $t$.

In weight allocation, grid power supply quality should be prioritized. Secondly, owners' participation should be encouraged. However, service costs of power grid shouldn't be too high. Last but not least, it is necessary to reduce power loss, namely the target priority is ranked as $f_{1}>f_{4}>f_{3}>f_{2}$

(2) Constraint Conditions

1) Equality constraints

Power flow constraint conditions must be satisfied. The power flow equation is applied to calculate node voltage and power grid loss, so the power flow constraint conditions must be satisfied.[10]

$$
\begin{aligned}
& \Delta P_{i}=P_{i}-U_{i} \sum_{j \in i} U_{j}\left(G_{i j} \cos \theta_{i j}+B_{i j} \sin \theta_{i j}\right) \\
& \Delta Q_{i}=Q_{i}-U_{i} \sum_{j \in i} U_{j}\left(G_{i j} \sin \theta_{i j}-B_{i j} \cos \theta_{i j}\right) \\
& i=1, \ldots, N_{2}
\end{aligned}
$$

In th formula: $P_{i}$ and $Q_{i}$ are active power and inactive power of the node $i$ respectively; $U_{i}$ is the voltage of node $i, G_{i j}$ and $B_{i j}$ stand for the conductivity susceptance between the nodes $i$ and $j ; \delta_{i j}$ represents the voltage phase angle difference between node $i$ and $j$.

2) Inequality constraints

(1) Limited by battery life protection, it is impossible to discharge all electricity in batteries, namely

$$
E \leq S O C-(1-D O D)
$$

In the formula: $E$ is the available electric quantity from electric vehicles; $S O C$ is surplus capacity of batteries, $D O D$ stands for discharge depth of batteries.

(2) When electric vehicles discharge, due to large loss in batteries, only to have the higher load rate in lines can electric vehicles discharge in power connection. After discharging, load rate of lines can't be too low, namely

$$
L_{l} \leq L(t) \leq L_{h}
$$


In the formula: ${ }^{L_{l}}$ and ${ }^{L_{h}}$ stand for the lowest and highest discharge load rates, respectively.

4. Information Release Rule

(1)The information which is released in the network platform by the background has two main parts, the demand information and the basic information.

(2)The specific information of electricity demand is charging and discharging plan. Distribute the electricity demand to client platform in fixed time. Collecting the users' feedback and feeding back the adjustable capacity to the power grid side within the fixed time.

(3)Release the electricity price forecasting information, the position of the charging pile and use condition to the platform real-timely. Guarantee the effectiveness of information as the basis of client choice.

\section{Work Process}

Deal with the data from power grid, such as loading forecasting, etc. Make charging and discharging plan.

Release the demand of electric vehicle discharge capacity and price information in web platform in the early time.

The owner decides whether they participate in discharging in the scheduled time.

Judge whether the owners have ability to discharge by detecting the electric vehicle battery parameters.

Feed back the charging and discharging plan to grid according to the participation situation which users choose.

The owners park the car around the charging pile in advance and connect the charging wire and then verify the order.

When time to, the platform will control the charging pile automatically and make the electric vehicle start charging.

\section{System Function}

(1)Power Bidirectional Exchange Function: Power can be transmitted to grid (electric vehicle discharge state) and also the power can be absorbed from grid (electric vehicle charge state).

(2)Bidirectional Communication Function: Instructions can be accepted and the power information can be sent remotely. Peak regulation instruction can be sent to the electric vehicle by the dispatching of power grid through the communication network within charge station. The response of the electric vehicle is monitored and recorded and through communication network the response is fed back to the charging station (backstage management system).

(3)Real-Time Control: It has the function of Real-time information feedback, real-time control, the real-time updated information and demand, the rational allocation of resources.

\section{Conclusion}

This paper discusses the system structure of orderly charging and discharging management of electric vehicle and dispatching system based on the internet, analyzes the composition and function of four main units which are network platform, charging pile, power grid side and client and realize the function of bidirectional communication, bidirectional power exchange, real-time update and regulation and control. This system can allow users to use handheld terminal to realize the functions of battery capacity status query, charging pile position navigation, charging and discharging forecasting and set independently and provide a platform for the interaction between owner and power grid. At the same time, in network manager data processing section, the V2G proposed during making charging and discharging plan in multi-objective and multi-constraints model of distribution application can deal with the interest of power grid and owner very well and also it can ensure the safe and economic operation of power grid and guide owner to participate in the net sevice actively. This system puts forward effective solution scheme for the question of dispatching 
and regulation of electric vehicle in peak load regulation and have a certain reference value of the implementation and popularization of $\mathrm{V} 2 \mathrm{G}$ policy.

\section{References}

[1] X.R. Gong, R. Liu and X.M. Qin: Internet Oriented Design and Application of Intelligent Charging System of Electric Vehicle, Electricity power construction,(2015),No.7,p.222.

[2] Z.M. Guo: The design and implementation of Electric vehicle charging stations real-time Internet electric energy metering system, Electronic world,(2016), No.20,p.81.

[3] J.Y. Yuan, X.D. Wang, D.Q. Wang and H.M. Wang: The Study of Electric Vehicle's AC Charging Pile Control System Based on PLC, Journal of Qingdao university (engineering technology edition),(2015), No.2,p.38.

[4] Q/CSG 11516.8-2010, Acceptance Specification of Electric Vehicle Charging Station and Charging Pile, Guangzhou: China southern power grid company ,2010:

[5] G. Wen, H.J. Shang, C.B. Zhu and R.G. Lu: The System Design of Electric Vehicle AC Charging Pile, Modern Electronic Technology,(2012), No.21,p.124.

[6] GB/T 20234.2 - 2011, The part 2 of Conduction Charging Connectioin Device of Electric Vehicle : AC charging interface, Beijing: China Standards Press, 2012:

[7] K. Xu, Z.A. Zhou, D.Y. Wu, W.B. Geng and X.D. Li: The Control System Design of Electric Vehicle AC Charging Pile, Journal of Henan Science and Technology University (natural sciences edition),(2016), No.3,p.47.

[8] T.R. Gong, T. Li and R. Liu: Internet Oriented Operation Analysis of Electric Vehicle Intelligent Charging System, Power supply and consumption,(2015), No.12,p.11.

[9] H.L. Li, X.M.Bai and W.Tan: The Study of Electric Vehicle Network Access Technology in the Application of Distribution Network, Proceedings of the CSEE,(2012), No.S1, p.22.

[10] G.Y. Li: Power System Analysis Basis (China Machine Press, China 2011.) 